\title{
Analysis of masking data applicable to subband coding
}

Satoshi Nagata, Makoto Iwata, Junji Okuda, Li Kuan Wen, Tsuyoshi Usagawa, and Masanao Ebata

Faculty of Engineering, Kumamoto University,

39-1 Kurokami 2-chome Kumamoto, 860 Japan

(Received 22 April 1993)

Keywords: Overestimated masking effect, Spectrum unmasked, Subband coding

PACS number: 43.66.Dc

\section{Introduction}

Subband coding ${ }^{1}$ is one of the compression method in audio signal, in which the broadband audio signal is divided into subbands on frequency region, and the signal in each subband is quantized with low bit rate. Recently a useful technique for low-bit-rate transmission using auditory masking effect is studied. In this technique the bit rate of each subband is determined depending on the masking threshold. Sounds with high quality can be obtained with lower bit rate by this method. However, only the masking data of sounds with longer duration are used in most cases of this method and the masking data for the sounds with the shorter duration are needed for this coding.

In this article, masking patterns are obtained as a function of the maskee duration in simultaneous masking and the characteristics of masking are analyzed on the view of the spectral distribution.

\section{Transient-masking characteristics}

Figure 1 shows the masking pattern of a single puretone masker which was obtained by us in the previous experiment. $^{2)}$ Masking data like this have already been presented by H. Fastl ${ }^{3)}$ et al. (1979) and others. But these are not available for the subband coding because the masking threshold varies with the masker and maskee duration. Therefore, it should be considered that the masking threshold is always changing when the audio signal is presented to the listener, so the masking factor that is used in subband coding must be changed. If they are not, masking is overestimated, hence the quality of sounds gets worse.

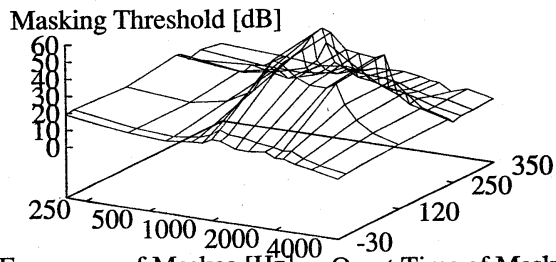

Frequency of Maskee $[\mathrm{Hz}]^{400}$ Onset Time of Maskee [ms]

Fig. 1 Masking pattern of a short tone masker. Masker duration: $250 \mathrm{~ms}$, masker frequency: $1000 \mathrm{~Hz}$, maskee duration: $10 \mathrm{~ms}$.

\section{Experimental method}

In the experiments, the method of limits is used. The stimulus tones, masker and maskee, are sinusoidal tones synthesized by a computer, which have the rise- and decay-time of $3.5 \mathrm{~ms} / 60 \mathrm{~dB}$. Figure 2 shows the time pattern of stimulus tones. The maskee is presented in the center of the masker, and its duration is varied from 10 to $160 \mathrm{~ms}$. The subjects were asked to listen to these tones in an anechoic room through headphones and respond to them at the changing point of sensation. An experimental condition are shown in the Table 1. Two male subjects (students) are participated in the experiment.

\section{Result and discussion}

When we use the masking data in subband coding, the compression ratio is improved as the amount of the masked area increases. However, the subjectively perceived quality degrades to the contrary. So we should look for the point that this trade-off is satisfied.

The results obtained by the two subjects showed almost same the tendency, so only results obtained by one subject are shown in the Fig. 3. The masking effect

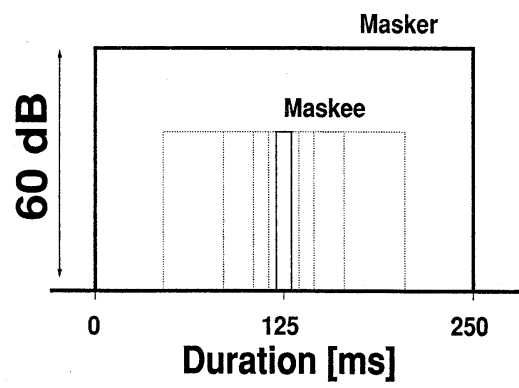

Fig. 2 Time course of the stimuli.

Table 1 Parameters used in the experiment.

\begin{tabular}{cc}
\hline Frequency $(\mathrm{Hz})$ & $250,500,1000,2000,4000$ \\
\hline $\begin{array}{c}\text { Masker duration }(\mathrm{ms}) \\
\text { Maskee duration }(\mathrm{ms})\end{array}$ & 250 \\
\hline
\end{tabular}




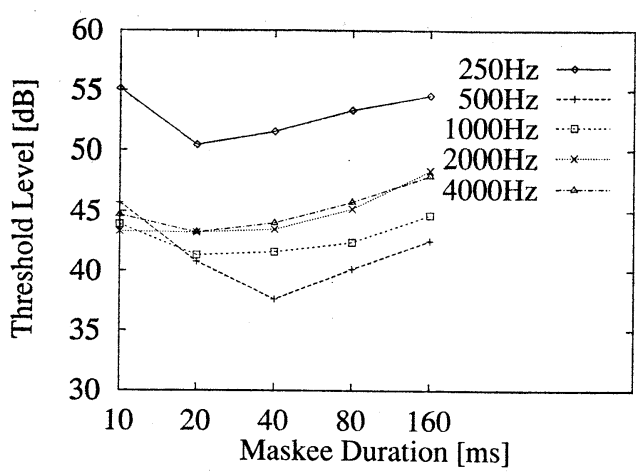

Fig. 3 Threshold Level of the tone bursts as a function of the duration. Parameter is frequency of the masker.

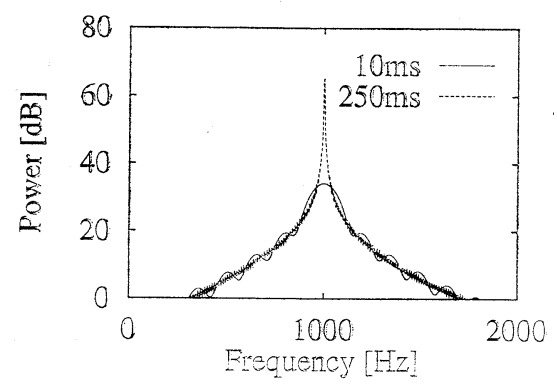

(a)

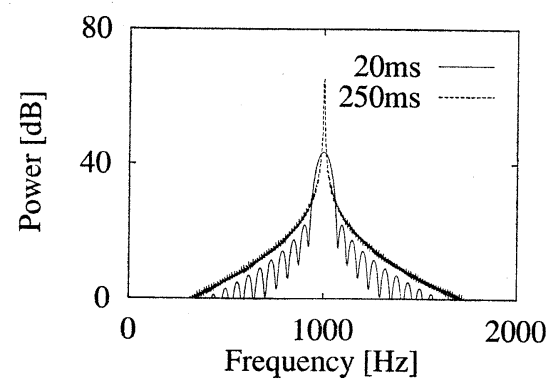

(b)

Fig. 4 Power spectrum of the short tone.

has its minimum value at $20 \sim 40 \mathrm{~ms}$ in maskee duration, and for the longer durations, the threshold increases again with the increases of maskee duration. As the duration of tone signal is made longer, its whole energy increases so that the threshold for the tone should become lower, but the result obtained is not the case. The reason is considered as follows; The maskee with the same frequency as the masker is difficult in

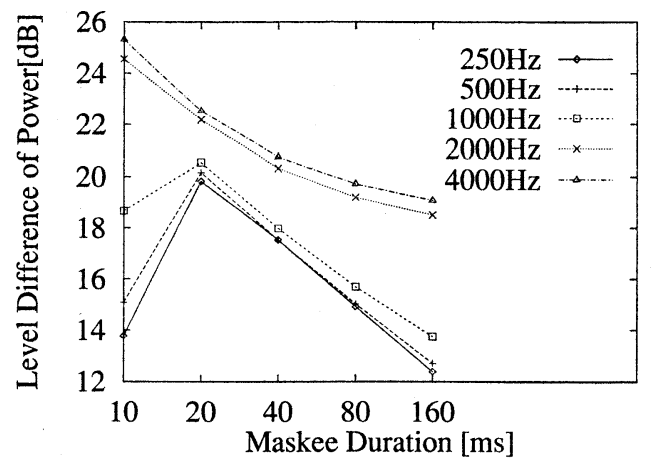

Fig. 5 The difference of spectral power between the masker and the maskee.

detection, but we can detect the maskee signal in the masker. The cue of detection may be the difference of the power spectrum between the masker and maskee. Figure 4 shows the spectra of the masker $(250 \mathrm{~ms}-$ duration) and maskee (10 ms in figure (a), and $20 \mathrm{~ms}$ in figure (b)). It is conceivable that we can detect the maskee based on the energy of the maskee unmasked by masker, so the spectrum unmasked is summed up and shown in Fig. 5. It is apparent that the spectral unmasked has its peak value at $20 \mathrm{~ms}$. At $2,000 \mathrm{~Hz}$ and $4,000 \mathrm{~Hz}$, however, the energy level decreases with duration of the maskee, and has no peak. The estimates for the two highest frequencies, in that they do not have any apparent peaks, are slight different from the experimental results. The discrepancy may depend on the number of wave included in the given duration.

As a result, we should choose the minimum value of the masking threshold data obtained in our experiment as the masking data for subband coding.

5. Conclusion

The masking experiment for short duration has done, and its data is analyzed for the purpose of using for subband coding.

\section{References}

1) J. W. Woods and S. D. O'Neil, "Subband coding of images," IEEE Trans. Acoust., Speech Signal Process. ASSP-34, 1278-1288 (1986).

2) M. Tagawa, A. Murakami, T. Nishimoto, T. Usagawa, and M. Ebata, "Effect of duration on temporal masking," Proc. Spring Meet. Acoust. Soc. Jpn., 371-372 (1992).

3) H. Fastl, "Temporal masking effects: III. Pure tone masker," Acustica 43, 282-294 (1979).

4) S. Namba, Handbook of Hearing (Nakanishiya Shuppan, Kyoto, 1984), Chap. 3 (in Japanese). 